\title{
Parathyroid Function in Primary Osteoporosis
}

\author{
B. Lawrence Riggs, Claude D. Arnaud, Jenifer Jowsey, \\ Ralph S. Goldsmith, and Patrick J. Kelly
}

From the Mayo Clinic and Mayo Foundation, Rochester, Minnesota 55901

\begin{abstract}
A B S T R A C T Two major species of serum immunoreactive parathyroid hormone (iPTH) were measured in 47 untreated patients with primary osteoporosis by using two highly specific radioimmunoassays. Mean iPTH was normal with one antiserum but was lower than normal $(P<0.001)$ with the other. iPTH values did not correlate with biochemical parameters or with the proportion of bone-resorbing surfaces in iliac crest bone biopsy specimens. These data suggest that the increased bone resorption is not due to increased parathyroid function in most osteoporotic patients. However, seven of our patients (15\%) appear to represent a separate population because they had increased values with one or the other of the antisera.
\end{abstract}

\section{INTRODUCTION}

Because of morphometric $(1,2)$ and biochemical evidence (3) that bone resorption is increased in primary osteoporosis and because it is believed that bone resorption is primarily regulated by parathyroid hormone (PTH), ${ }^{1}$ PTH may play a causal or permissive role in the pathogenesis of osteoporosis (4-7). However, parathyroid function has not been assessed directly in this disorder.

We measured serum immunoreactive $\mathrm{PTH}$ concentrations (iPTH) in normal and in osteoporotic persons to determine if parathyroid function is abnormal in osteoporosis.

\section{METHODS}

We studied 47 ambulatory patients with progressive osteoporosis of sufficient severity to produce crush fractures of the spinal column. The 43 women and four men in the group had a mean age of $64.9 \mathrm{yr}$ (range, 47-79 yr). No

Received for publication 26 June 1972 and in revised form 19 September 1972.

${ }^{1}$ Abbreviations used in this paper: $\mathrm{CH}-14 \mathrm{M}$, chicken antibovine-PTH antiserum; GP-1M, guinea pig anti-porcinePTH antiserum; PTH, parathyroid hormone; iPTH, immunoreactive PTH. patient had any evident medical disease that might produce osteoporosis and none had any clinically evident condition that might impair calcium absorption, such as liver disease, previous subtotal gastrectomy, or sprue. All had normal serum values for calcium, phosphorus, and alkaline phosphatase. Formal studies of intestinal absorption were not made. iPTH was determined in these patients prior to the initiation of any form of treatment.

The patients remained on their habitual diets; blood for serum iPTH determinations was drawn at 8:00 a.m. after an overnight fast. On the same serum sample, calcium was determined by atomic absorption spectrophotometry and phosphorus and alkaline phosphatase, by standard methods. Bone-forming and bone-resorbing surfaces in an iliac crest biopsy sample were determined by quantitative microradiography (1) in 27 of these patients.

For control values, serum iPTH and calcium were also determined in 146 normals, none of whom had a history of back pain ( 84 women and 62 men with ages distributed evenly between 20 and $78 \mathrm{yr}$ ); these values include previously reported data from 52 normal persons (8). Bone densitometry values (9) of the radius, obtained for twothirds of the normals more than $50 \mathrm{yr}$ old, were within the normal range for their age.

iPTH was measured by radioimmunoassay using two different antisera (GP-1M and $\mathrm{CH}-14 \mathrm{M})$. The first assay system used a guinea pig anti-porcine-PTH antiserum (GP$1 \mathrm{M}$ ) and has been described in detail previously (8). It measures iPTH in greater than $95 \%$ of normal sera. Sera from all patients studied were assayed in this system. The second assay system used a chicken anti-bovine-PTH antiserum $(\mathrm{CH}-14 \mathrm{M})$. This assay system measures iPTH in greater than $90 \%$ of normal sera. The radioimmunoassay was performed identically except that it was used at a higher concentration (1:3,000, compared with $1: 100,000$ for GP-1M) and that approximately five times less ${ }^{121} \mathrm{I}$ labeled bovine PTH was added to the incubation mixtures $(3,000 \mathrm{cpm}$ for $\mathrm{CH}-14 \mathrm{M}$ compared with $15,000 \mathrm{cpm}$ for GP-1M). Incubation mixtures with $\mathrm{CH}-14 \mathrm{M}$ were counted for sufficient times to record 10,000 counts. Standard curves with $\mathrm{CH}-14 \mathrm{M}$ antiserum using both purified human PTH from hyperparathyroid serum and a standard preparation of human PTH obtained from the media of cultured parathyroid adenoma explants have been published previously $(10,11)$. Sera of 20 of the osteoporotic patients and 36 normals (17 more than $50 \mathrm{yr}$ old) were assayed for iPTH using $\mathrm{CH}-14 \mathrm{M}$ antiserum. In both assays, the criteria for a measurable iPTH value were those previously published (8). Human hyperparathyroid serum was used as a refer- 


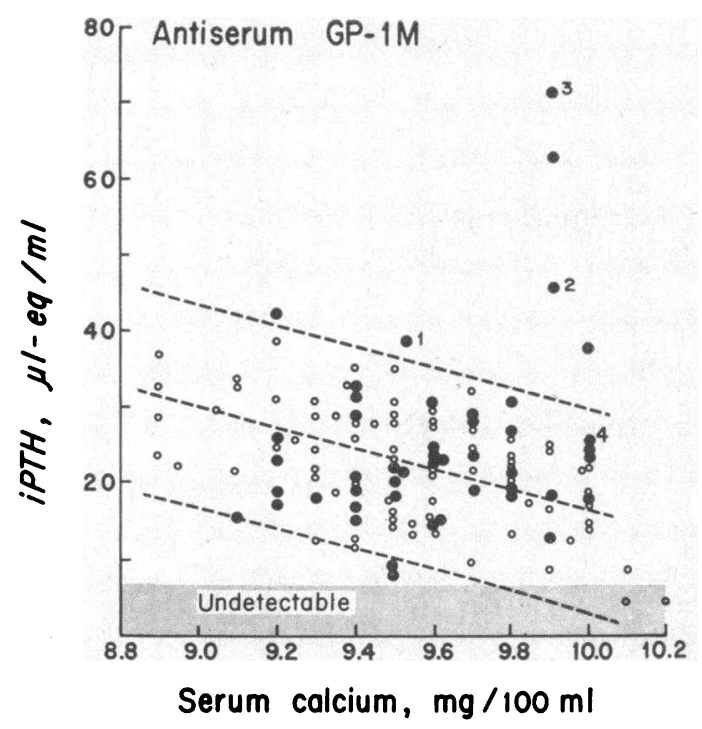

Figure 1 Serum iPTH (antiserum GP-1M) as function of serum calcium. Individual values for normals over age $50 \mathrm{yr}$ are given by $(O)$; regression line and $95 \%$ confidence limits are given by broken lines. Individual values for osteoporotic patients are given by $(\bullet)$. Patients designated by numbers 1,2 , and 3 underwent intravenous calcium infusions; iPTH was not suppressed in patients 1 and 2 but was suppressed in patient 3 . Patient 4 had high iPTH values with antiserum $\mathrm{CH}-14 \mathrm{M}$.

ence standard in both assays and the coefficient of interassay variation for replicate determinations was $12-14 \%$.

Two major molecular species of PTH have been previously demonstrated in serum of patients with primary hyperparathyroidism $(12,13)$. The two antisera used in the present study have markedly different specificities for these two species. Gel-filtration studies of hyperparathyroid sera ${ }^{2}$ have demonstrated that antiserum GP-1M predominantly measures $\mathrm{PTH}$ immunoreactivity in fractions eluting in a region corresponding to mol wt 7000 whereas antiserum $\mathrm{CH}-14 \mathrm{M}$ predominantly measures immunoreactivity eluting in the region of mol wt 9500 . The normal range for iPTH is undetectable to $40 \mu \mathrm{l}-\mathrm{eq} / \mathrm{ml}$ measured with GP-1M and undetectable to $250 \mu \mathrm{l}-\mathrm{eq} / \mathrm{ml}$ with $\mathrm{CH}-14 \mathrm{M}$. The difference in the normal ranges obtained with these two antisera probably relates to $(a)$ differences in the specificities of the antisera for the 9500 and $7000 \mathrm{~mol}$ wt species of PTH in hyperparathyroid sera and (b) apparent differences in the concentration of these species in the standard hyperparathyroid reference serum. From our studies, ${ }^{2}$ there appears to be much less $9500 \mathrm{~mol}$ wt than $7000 \mathrm{~mol} \mathrm{wt}$ species of iPTH in hyperparathyroid serum. Therefore, since $\mathrm{CH}-14 \mathrm{M}$ antiserum is relatively specific for the $9500 \mathrm{~mol} \mathrm{wt}$ species of iPTH, it would require proportionally more standard hyperparathyroid serum to achieve the same inhibition of the immune reaction than with GP-1M.

\section{RESULTS}

iPTH values obtained with antiserum GP-1M. $\mathrm{iPTH}$ was measurable in all but four sera from normals;

${ }^{2}$ Arnaud, C. D. Unpublished data.

Riggs, Arnaud, Jowsey, Goldsmith, and Kelly values did not differ significantly between men and women. There was a small positive regression of iPTH on age $(r=0.186, P<0.05)$. As previously reported (8), there was an inverse correlation of iPTH with serum calcium concentration $(r=-0.53, \quad P$ $<0.001$ ). For 79 normals more than $50 \mathrm{yr}$ old, mean iPTH ( \pm SE) was $22.4 \pm 0.9 \mu 1-\mathrm{eq} / \mathrm{ml}$.

For patients with osteoporosis, mean iPTH ( \pm SE) was $25.1 \pm 1.8 \mu 1-\mathrm{eq} / \mathrm{ml}$ (range, 9.0-71.2), not significantly different from that of normals more than $50 \mathrm{yr}$ old. The serum calcium values did not differ significantly in the two groups. There was no significant correlation of iPTH with serum calcium concentration (Fig. 1) or with serum phosphorus or alkaline phosphatase or, in the 27 patients who had iliac crest biopsies, with the proportion of surfaces undergoing formation or resorption.

Six of the 47 osteoporotic patients had iPTH values that were above the 97.5 percentile limit for the regression of iPTH on serum calcium concentration in normals. Three of these six patients subsequently underwent an intravenous infusion of calcium (as the glucoheptonate, $4 \mathrm{mg} / \mathrm{kg}$ per $\mathrm{h}$ for $4 \mathrm{~h}$ in two and $8 \mathrm{~h}$ in one); at this time all three were receiving treatment for their osteoporosis (calcium supplements and calcitonin for 6 months in two and phosphate supplements for 3 months in one). iPTH remained unchanged or increased slightly during the $8 \mathrm{~h}$ after the beginning of the infusion in two patients; it decreased from 71 to $11 \mu \mathrm{l}-\mathrm{eq} / \mathrm{ml}$ in one (Fig. 2). In four normals who received a $4 \mathrm{~h}$ infusion of calcium at the same rate, iPTH was undetectable by $2 \mathrm{~h}$ and remained so through $12 \mathrm{~h}(8)$.

iPTH values obtained with antiserum CH-14M. iPTH was measurable in all but two sera from normals; values did not differ significantly between men and women or between individuals over and under age

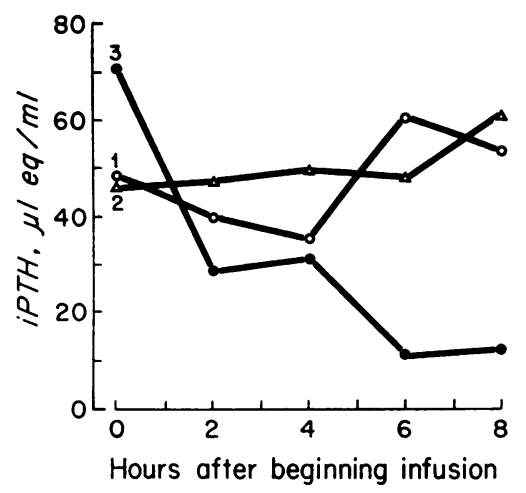

FIGURE 2 iPTH values in three patients who received intravenous calcium infusions $(4 \mathrm{mg} / \mathrm{kg}$ per $\mathrm{h}$ for $4 \mathrm{~h}$ in patients 1 and 2 and for $8 \mathrm{~h}$ in patient 3 ). Designation of patients is as in Fig. 1. 
$50 \mathrm{yr}$. For these 37 normals, mean iPTH ( $\pm \mathrm{SE}$ ) was $127.2 \pm 9.2 \mu \mathrm{l}-\mathrm{eq} / \mathrm{ml}$. For the 20 osteoporotic patients who also had values determined with this antiserum, mean iPTH ( \pm SE) was $69: 9 \pm 14.2 \mu \mathrm{l}-\mathrm{eq} / \mathrm{ml}$, significantly lower $(P<0.001)$ than normal. However, one patient had a value higher than normal. In neither normals nor osteoporotic patients was there a significant correlation between iPTH and serum calcium concentration (Fig. 3).

\section{DISCUSSION}

iPTH values were not increased in the majority of osteoporotic patients when the assay was based on antiserum GP-1M but they were significantly lower than normal when assayed with antiserum CH-14M. Furthermore, if PTH excess is the sole cause of increased bone loss in osteoporosis, a correlation between iPTH values and the amount of bone-resorbing surface would be expected; this was not found. However, in another study which also used antiserum GP-1M, iPTH values correlated directly with bone-resorbing surface when secondary hyperparathyroidism was induced in osteoporotic patients by chronic therapy with porcine calcitonin (14). Also, iPTH values by either antiserum correlated directly with the osteoclast count and with osteocytic osteolysis in bone from patients with primary hyperparathyroidism..$^{3}$ Although it is possible that PTH may play a permissive role, our findings suggest that increased parathyroid function is not a distinguishing feature of most osteoporotic patients and that there may be a partial inhibition of PTH secretion, as previously suggested by us (15).

It is not entirely clear why different results were obtained with the two antisera in the osteoporotic patients. The fraction of iPTH (mol wt 9500) which $\mathrm{CH}-14 \mathrm{M}$ measures primarily appears to be the principal secretory product of parathyroid glands in vivo (13); this form of iPTH apparently is converted peripherally into a smaller molecule ( $\mathrm{mol} \mathrm{wt} \sim 7000$ ) (13) which GP-1M measures primarily. Our finding that iPTH assayed with $\mathrm{CH}-14 \mathrm{M}$ is lower than normal and $\mathrm{iPTH}$ assayed with GP-1M is normal might indicate decreased PTH secretion coupled with decreased rate of metabolism of the lower molecular weight form of the hormone.

The data also suggest that there are two populations of osteoporotic patients-a large majority with normal or decreased parathyroid function and a small minority with increased parathyroid function. The presence of two types of parathyroid function in osteoporosis might explain the failure to observe the expected inverse correlation between iPTH (assayed with antiserum GP$1 \mathrm{M}$ ) and serum calcium concentration. Of the seven

\footnotetext{
${ }^{3}$ Bordier, P., and C. D. Arnaud. Unpublished data.
}

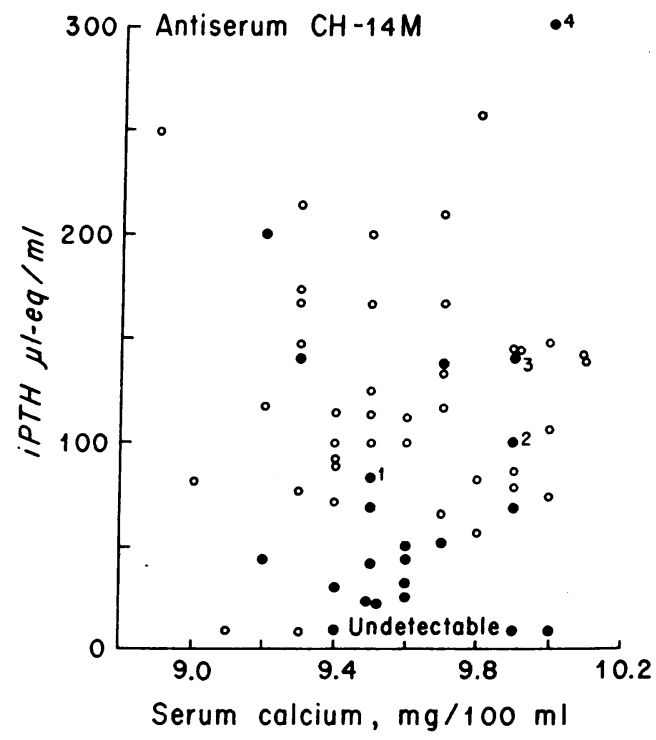

Figure 3 Serum iPTH (antiserum $\mathrm{CH}-14 \mathrm{M}$ ) as a function of serum calcium, in normals $(O)$ and in 20 osteoporotic patients $(\bullet)$. Designation of patients $1-4$ is the same as in Fig. 1. Patients 1,2 , and 3 were the only patients reassayed with antiserum $\mathrm{CH}-14 \mathrm{M}$ who had higher than normal iPTH values with antiserum GP-1M.

patients with increased $\mathrm{iPTH}$ values ( $\mathrm{six}$ with antiserum GP-1M and one with antiserum $\mathrm{CH}-14 \mathrm{M}$ ), five had serum calcium concentrations in the high normal range and it is possible that they represent examples of normocalcemic primary hyperparathyroidism. This possibility is particularly likely in the two patients whose iPTH values were not suppressed by intravenous calcium infusion, although prior treatment may have influenced these results. That more patients had increased values for $\mathrm{iPTH}$ with antiserum GP-1M than with $\mathrm{CH}-14 \mathrm{M}$ is consistent with the greater ability of the former antiserum to separate normals from those with primary hyperparathyroidism (16). An alternative possibility is that increased iPTH in these seven patients was due to secondary hyperparathyroidism resulting from occult intestinal malabsorption of calcium.

\section{ACKNOWLEDGMENTS}

This investigation was supported in part by Research Grants AM-8658, AM-12302, and RR-585 from the National Institutes of Health, U. S. Public Health Service.

We appreciate the excellent technical assistance of Mrs. Judith A. Larsen, Miss Mary Jo Bill, and Mrs. Karen J. Laakso.

\section{REFERENCES}

1. Jowsey, J.. P. J. Kelly, B. L. Riggs, A. J. Bianco, Jr., D. A. Scholz, and J. Gershon-Cohen. 1965. Quantitative microradiographic studies of normal and osteoporotic bone. J. Bone Jt. Surg. Am. Vol. 47 : 785.

2. Wu, K., S. Jett, and H. M. Frost. 1967. Bone resorp- 
tion rates in rib in physiological, senile, and postmenopausal osteoporosis. J. Lab. Clin. Med. 69: 810.

3. Flanagan, B., S. Ault, and G. Nichols, Jr. 1970. Bone metabolism in osteoporosis. In Osteoporosis. U. S. Barzel, editor. Grune \& Stratton, Inc., New York. 217.

4. Hossain, M., D. A. Smith, and B. E. C. Nordin. 1970. Parathyroid activity and postmenopausal osteoporosis. Lancet. 1: 809.

5. Nordin, B. E. C. 1971. Clinical significance and pathogenesis of osteoporosis. Br. Med. J. 1: 571 .

6. Jowsey, J., and P. Balasubramaniam. 1972. Effect of phosphate supplements on soft-tissue calcification and bone turnover. Clin. Sci. (Oxf.). 42: 289.

7. Bordier, P. J., and S. Tun-Chot. 1972. Quantitative histology of metabolic bone disease. Clin. Endocrinol. $1: 197$.

8. Arnaud, C. D., H. S. Tsao, and T. Littledike. 1971. Radioimmunoassay of human parathyroid hormone in serum. J. Clin. Invest. 50: 21.

9. Cameron, J. R., and J. Sorenson. 1963. Measurement of bone mineral in vivo: an improved method. Science (Wash. D. C.). $142: 230$.

10. Arnaud, C. D., H. S. Tsao, and S. B. Oldham. 1970. Native human parathyroid hormone: an immunochemical investigation. Proc. Natl. Acad. Sci. U. S. A. 67 : 415.
11. Arnaud, C. D., G. W. Sizemore, S. B. Oldham, J. A. Fischer, H. S. Tsao, and E. T. Littledike. 1971. Human parathyroid hormone: glandular and secreted molecular species. Am. J. Med. 50: 630.

12. Canterbury, J. M., and E. Reiss. 1971. Fractionation of circulating parathyroid hormone (PTH) in man. J. Lab. Clin. Med. 78: 814. (Abstr.)

13. Habener, J. F., D. Powell, T. M. Murray, G. P. Mayer, and J. T. Potts, Jr., 1971. Parathyroid hormone: secretion and metabolism in vivo. Proc. Natl. Acad. Sci. U. S. A. $68: 2986$.

14. Jowsey, J., B. L. Riggs, R. S. Goldsmith, P. J. Kelly, and C. D. Arnaud. 1971. Effects of prolonged administration of porcine calcitonin in postmenopausal osteoporosis. J. Clin. Endocrinol. Metab. 33: 752.

15. Kelly, P. J., J. Jowsey, B. L. Riggs, and L. R. Elveback. 1967. Relationship between serum phosphate concentration and bone resorption in osteoporosis. J. Lab. Clin. Med. 69 : 110.

16. Arnaud, C., R. Goldsmith, J. Bischoff, G. Sizemore, S. Oldham, and J. Larson. 1972. Antibody specificity, immunochemical heterogeneity, and the interpretation of measurements of plasma parathyroid hormone by radioimmunoassay. J. Clin. Invest. 51: 5a. (Abstr.) 